\title{
Recombinant adeno-associated virus-, polyethylenimine/plasmid- and lipofectamine/carboxyfluorescein-labeled small interfering RNA-based transfection in retinal pigment epithelial cells with ultrasound and/or SonoVue
}

\author{
HONGLI LI, CAIFENG WAN and FENGHUA LI
}

Department of Ultrasound, Ren Ji Hospital, School of Medicine, Shanghai Jiao Tong University, Shanghai 200127, P.R. China

Received February 8, 2014; Accepted December 19, 2014

DOI: $10.3892 / \mathrm{mmr} .2015 .3219$

\begin{abstract}
The present study was conducted to investigate the efficacy and safety of ultrasound (US)-mediated transfection of the type 2 recombinant adeno-associated virus (AAV) vectors encoding the enhanced green fluorescent protein (EGFP) gene (rAAV), polyethylenimine (PEI)/plasmid EGFP-N1 (pDNA) or lipofectamine (L)/carboxyfluorescein (FAM)-labeled small interfering RNA (siRNA) in the human ARPE-19 retinal pigment epithelial (RPE) cell line, with or without the addition of SonoVue. Cultured RPE cells were exposed to US, with or without SonoVue under different conditions, including variation in the intensity and duration of treatment, and the dose of microbubbles. The effects of ultrasound-targeted microbubble destruction (UTMD) on the structure of pDNA and the transfection ability of rAAV, PEI/pDNA and L/siRNA were also evaluated. Furthermore, the effect of UTMD on RPE cells was evaluated at 0 and $24 \mathrm{~h}$ following UTMD. US-mediated transfection (USMT) significantly increased L/siRNA transfection efficiency, as measured by the transgene expression per cell and the percentage of transfected cells. UTMD significantly increased rAAV and PEI/pDNA transfer to RPE cells. UTMD-mediated rAAV or PEI/pDNA delivery was more effective than USMT-mediated delivery of siRNA. Evaluating cell viability at $24 \mathrm{~h}$ post-UTMD provided more valuable information than immediate evaluation following UTMD. Furthermore, there was minimal cytotoxicity and minimal change to the structure of pDNA under the optimal parameters. UTMD/US may be of use in enhancing rAAV, PEI/pDNA and L/siRNA transgene expression of ARPE-19 cells in vitro. Studies on the transfection of different nucleotides (such as pDNA and siRNA) and different types of vectors (chemical and biological)
\end{abstract}

Correspondence to: Professor Fenghua Li, Department of Ultrasound, Ren Ji Hospital, School of Medicine, Shanghai Jiao Tong University, 1630 Dongfang Road, Shanghai 200127, P.R. China E-mail: fenghualipro@163.com

Key words: ultrasound, SonoVue, recombinant adeno-associated virus, polyethylenimine, lipofectamine mediated by UTMD may provide useful information to guide future in vivo and transfection studies.

\section{Introduction}

A variety of genetic diseases of the retina, including retinitis pigmentosa, leber congenital amaurosis and proliferative vitreoretinopathy are associated with disorder of retinal pigment epithelial (RPE) cells. To date, no satisfactory treatments for these disorders have been identified. Gene therapy is a promising therapeutic option for these diseases. RPE cells are a potential target for treatment with gene delivery, since they are able to secrete transgenes to the neural retina following in vivo transfection and they may be transfected in vitro and then re-implanted into the eye $(1,2)$. Non-viral gene delivery to RPE cells has shown limited success due to its relatively low efficiency. RPE cells are a type of cell which is difficult to transfect (3-6). Polyethylenimine (PEI), which is considered to be the gold standard polymeric transfection reagent, only achieved $8 \%$ transfection efficiency in APRE-19 cells in a previous study (3). Thus, the major challenge is to identify an effective delivery system with improved performance in RPE cells.

In 1987, Fechheimer et al (7) proposed that ultrasound may promote gene transfection in vitro. Ultrasound-targeted microbubble destruction (UTMD) has been shown to be safe and efficient for use in various organs. However, the mechanisms by which US-mediated transfection (USMT) or UTMD enhance gene transfection have yet to be elucidated. Sonoporation is considered to be the principal factor. In the field of ophthalmology, a number of studies have investigated the effects of USMT or UTMD on the cornea. However, there are far fewer reports on these treatments in the retina (8-12). Despite previous studies by this group on UTMD-mediated recombinant adeno-associated virus and plasmidDNA delivery to APRE-19 cells (12-14), there is little information on the effect of UTMD in RPE cells (8). A previous study showed that UTMD increased recombinant adeno-associated virus transfection efficiency to APRE-19 cells by $\sim 75 \%$ with $97.38 \pm 3.16 \%$ cell viability (12). Nearly all studies on UTMD and gene transfer have evaluated cell viability in response to different techniques and at various time-points (7-14). However, the optimal time at which to accurately assess the effect of UTMD on cell viability is yet to be 
determined. Therefore, in the present study, cell viability at 0 and $24 \mathrm{~h}$ following UTMD, as well as the effectiveness of UTMD in promoting type 2 recombinant adeno-associated virus vector encoding the enhanced green fluorescence protein (EGFP) gene (rAAV), polyethylenimine (PEI)/plasmid (p)DNA and lipofectamine (L)/carboxyfluorescein (FAM)-labeled siRNA (L/siRNA) transfection, were studied. Studies on different genes (including pDNA and siRNA) and in different types of vectors (chemical and biological) mediated by UTMD may provide useful information to guide future developments in this field.

\section{Materials and methods}

Cell culture. The human ARPE-19 RPE cell line was obtained from the American Type Culture Collection (CRL-2302, Rockville, MD, USA). Cells were cultured 1:1 in Dulbecco's modified Eagle's medium (DMEM)/F12 with 10\% fetal bovine serum, $100 \mathrm{U} / \mathrm{ml}$ penicillin and $100 \mu \mathrm{g} / \mathrm{ml}$ streptomycin (Gibco Laboratories, Gaithersburg, MD, USA) for the rAAV and PEI/pDNA transfections, and in medium without penicillin for the siRNA transfection. RPE cells were placed into every other well of 24-well plates, at a concentration of $2 \times 10^{5}$ cells per well prior to infection.

\section{Ultrasound contrast agent, rAAV, PEI/pDNA and L/siRNA}

SonoVue microbubble (MB) contrast agent. SonoVue microbubble contrast agent (Bracco, Milan, Italy) was reconstituted in $5 \mathrm{ml}$ saline solution according to the manufacturer's instructions. SonoVue microbubbles are lipid shells filled with sulfur hexafluoride gas, containing $\sim 2-5 \times 10^{8}$ microbubbles $/ \mathrm{ml}$ and with an average diameter of 2.5-6.0 $\mu \mathrm{m}$. A quantity of $2 \times 10^{8}$ $\mathrm{MBs} / \mathrm{ml}$ was taken as a basis for calculating the $\mathrm{MB} / \mathrm{cell}$ ratio.

$r A A V 2$. rAAV2-EGFP $\left[1 \times 10^{12}\right.$ vector genomes $\left.(\mathrm{vg}) / \mathrm{ml}\right]$ was obtained from Vector Gene Technology Co., Ltd. (Beijing, China). A dose of $1 \times 10^{3}$ MOI (multiplicity of infection; vector genomes/number of cells) was used.

siRNA.FAM-labeled siRNA was obtained from GenePharma Co., Ltd. (Shanghai, China). The concentration used was $20 \mathrm{nM}$. Lipofectamine $^{\text {TM }} 2000$ was purchased from Invitrogen Life Technologies (Carlsbad, CA, USA). L ( $1 \mu \mathrm{l} /$ well) was diluted with $50 \mu \mathrm{l}$ opti-minimum essential medium (MEM) I Reduced Serum medium (Gibco Laboratories) and incubated at room temperature for $5 \mathrm{~min}$. siRNA ( $1 \mu \mathrm{l} /$ well) was diluted with $25 \mu \mathrm{l}$ opti-MEMI Reduced Serum medium. Incubated diluted L was mixed with diluted siRNA and maintained at room temperature for $20 \mathrm{~min}$ in order to obtain the L/siRNA mixture.

Branched PEI. PEI (molecular weight, $25 \mathrm{kDa}$ ) was obtained from Sigma-Aldrich (St. Louis, MO, USA). The 4.7-kb plasmid EGFP-N1 $(1 \mu \mathrm{g} / \mu \mathrm{l})$ was extracted and identified according to the manufacturer's instructions (Plasmid Maxi Preparation kit; Qiagen, Valencia, CA, USA). An N/P ratio of 8 and a pDNA concentration of $1 \mu \mathrm{g} /$ cell was used. This was maintained for $20 \mathrm{~min}$ at room temperature once PEI was mixed with pDNA.

Cell damage assay. RPE cells were seeded into 48 wells of a 96-well plate (with four adjacent wells as a group separated by gaps of four blank wells) at a concentration of $1 \times 10^{5}$ cells per well. Cells were exposed to US with various parameters (frequency, $1 \mathrm{MHz}$; intensity, 1, 2 or $3 \mathrm{~W} / \mathrm{cm}^{2}$; duration, 60 or
$120 \mathrm{sec}$; duty cycle, 20,50 or $100 \%$ ) with or without SonoVue (MB/cell ratio, 20:1, 50:1 or 70:1). The final volume per well during exposure to US was $100 \mu \mathrm{l}$, including DMEM and SonoVue. At 0 and $24 \mathrm{~h}$ following administration of UTMD with various exposure parameters, tryptan blue (Invitrogen Life Technologies) and MTS assays (AQueous One Solution Cell Proliferation Assay kit; Promega Corp., Madison, WI, USA) were performed.

Ultrasound exposure protocol. Cells were seeded onto a 24-well plate at a volume of $200 \mu \mathrm{l}$ per well during exposure to US, including DMEM, rAAV, PEI/pDNA and L/siRNA. In order to attach $\mathrm{rAAV}, \mathrm{PEI} / \mathrm{pDNA}$ or L/siRNA to MBs, the mixture was incubated at room temperature for $5 \mathrm{~min}$, with or without SonoVue (MB/cell ratio, 50:1), to allow for the development of an electrostatic attachment. The mixed solution was added to the plates which were then exposed to US. At $2 \mathrm{~h}$ post-infection, the plates were supplemented with DMEM to achieve a final volume of $500 \mu \mathrm{l}$. At $12 \mathrm{~h}$ following infection, the solution was replaced with fresh DMEM.

A therapeutic US machine (Topteam161; Chattanooga Medical Supply, Inc., Chattanooga, TN, USA) was used as previously described (12). The following experimental conditions were used for the transfection experiments: Frequency, $1 \mathrm{MHz}$; US intensity, $1 \mathrm{~W} / \mathrm{cm}^{2}$; duration, $60 \mathrm{sec}$; pulse wave with $50 \%$ duty cycle; SonoVue dose, negative; and MB/cell ratio, 50:1. RPE cells were infected by $\mathrm{rAAV}, \mathrm{PEI} / \mathrm{pDNA}$ and L/siRNA alone or in combination with US, SonoVue or UTMD treatment. Cells infected by rAAV, PEI/pDNA and L/siRNA alone served as control groups.

Gene transfer efficiency. At $48 \mathrm{~h}$ following rAAV and $\mathrm{PEI} / \mathrm{pDNA}$ transfection, and at $12 \mathrm{~h}$ following L/siRNA transfection, EGFP or FAM expression was observed and images were captured using inverted fluorescence microscopy (Zeiss Axiovert S100; Zeiss, Oberkochen, Germany). The percentage of infected cells and the mean fluorescence density were examined by fluorescence-activated cell sorting (EPICS XL; Beckman Coulter, Miami, FL,USA).

Effect of UTMD on pDNA, rAAV, PEI/pDNA and L/siRNA. The effect of UTMD on the structure of pDNA in the supernatant was evaluated by agarose gel electrophoresis. The effects of UTMD parameters on rAAV, PEI/pDNA and L/siRNA were evaluated by assessing the transfection efficiency of the cells following UTMD. Medium (200 $\mu \mathrm{l})$ containing rAAV, $\mathrm{PEI} / \mathrm{pDNA}$ or L/siRNA only was exposed to UTMD and the percentage of infected cells was then evaluated. The UTMD conditions used were: $1 \mathrm{~W} / \mathrm{cm}^{2} ; 50 \%$ duty cycle; $60 \mathrm{sec}$; and $\mathrm{MB} /$ cell ratio, 50:1.

Statistical analysis. Values are expressed as the mean \pm standard deviation. Cell viability conditions were performed in twelve parallel wells. All transfection conditions were performed in three parallel wells, following identical procedure and repeated thrice. For data following a Gaussian (normal) distribution, an unpaired Student's t-test was used to determine the significance of the difference between two groups. If not, a nonparametric Mann-Whitney test was used. One way analysis of variance was employed to determine the 
A

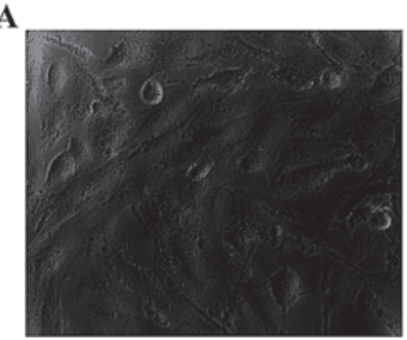

$\mathbf{E}$

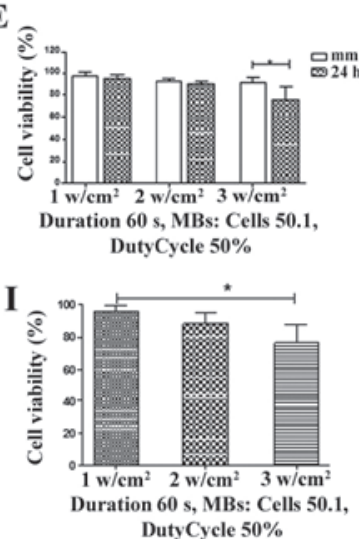

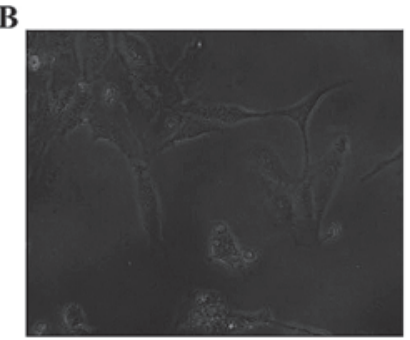

F
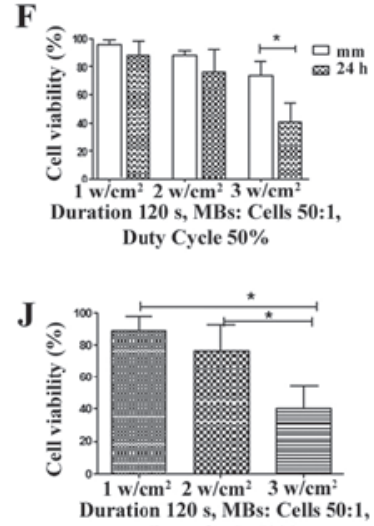
Duty Cycle 50\%

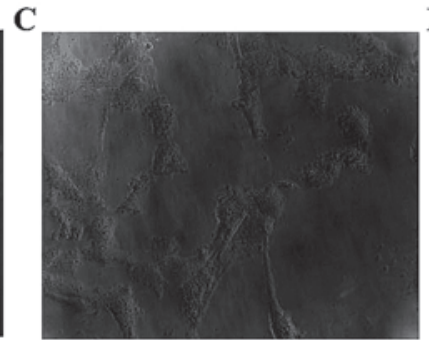

G

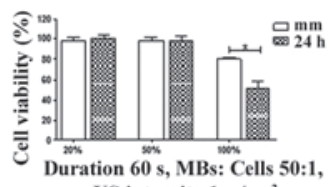

US intensity $1 \mathrm{w} / \mathrm{cm}^{2}$

K

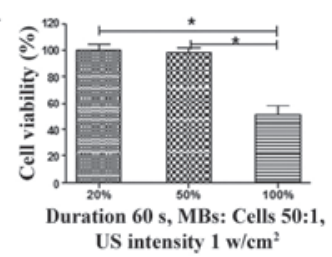

D

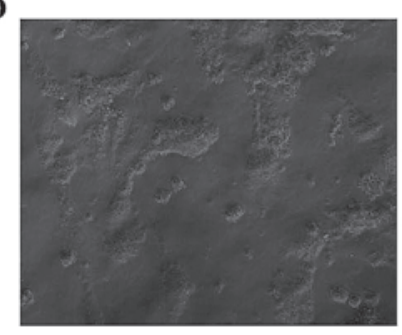

H

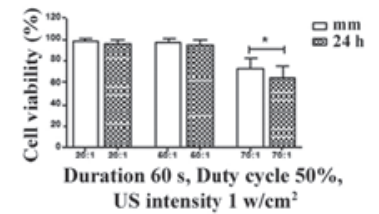

L

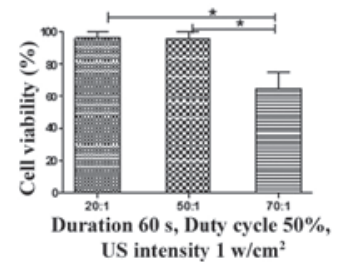

Figure 1. Effect of different UTMD conditions on RPE cell viability. (A) Untreated RPE cells were observed in adherent monolayers. (B-D) With increasing energy, cellular morphology turned round or irregular with increasing numbers of floating cells or blank areas; (D) Following exposure to a combination of upper limit parameters, cellular morphology was irregular and the cytoplasm showed poor refractivity (magnification, $\mathrm{x} 400$ ). (E-H) Cell viability at 0 and $24 \mathrm{~h}$ following UTMD with changes in (E) US intensity at a duration of $60 \mathrm{sec},(\mathrm{F}) \mathrm{US}$ intensity at a duration of $120 \mathrm{sec},(\mathrm{G})$ duty cycle and (H) MB/cell ratio. (I-L) Cell viability at $24 \mathrm{~h}$ following UTMD with changes in (I) US intensity at a duration of $60 \mathrm{sec}$, (J) US intensity at a duration of $120 \mathrm{sec}$, (K) duty cycle and (L) MB/cell ratio. ${ }^{*} \mathrm{P}<0.05$ between indicated values. UTMD, ultrasound-targeted microbubble destruction; RPE, retinal pigment epithelial cells; US, ultrasound; MB, microbubble.

significance of the differences in multiple comparisons. $\mathrm{P}<0.05$ was considered to indicate a statistically significant difference. The software package used was GraphPad Prism, version 5.0 (GraphPad Software, Inc., La Jolla, CA, USA).

\section{Results}

Comparison of cell viability 24 host-UTMD with various exposure parameters. As shown in Fig. 1A-D, the effects of various UTMD conditions on RPE cell morphology were observed using a light microscope. Untreated RPE cells presented an adherent monolayer shape (Fig. 1A), and following an increase in energy, the cellular morphology turned round or irregular (Fig. 1B-D). In addition, under the combination of upper limit parameters, cellular morphology turned irregular and the cytoplasm showed poor refractivity with a ground glass appearance (Fig. 1D).

\section{Cell viability assays}

Comparison of cell viability at 0 and 24 following UTMD.

US intensity and exposure duration. US intensity was varied between 1, 2 and $3 \mathrm{~W} / \mathrm{cm}^{2}$. The duration of exposure was varied between 60 and $120 \mathrm{sec}$. The MB/cell ratio was $50: 1$ and the duty cycle was $50 \%$. Cell viability at $24 \mathrm{~h}$ was lower than that immediately post-UTMD in all subgroups, although the difference was not significant at 1 or $2 \mathrm{~W} / \mathrm{cm}^{2}$. At an US intensity of $3 \mathrm{~W} / \mathrm{cm}^{2}$ and a duration of $60 \mathrm{or} 120 \mathrm{sec}$, the cell viability was significantly reduced at $24 \mathrm{~h}$ compared with that at $0 \mathrm{~h}(\mathrm{P}=0.0021$ and 0.019 , respectively; Fig. $1 \mathrm{E}$ and $\mathrm{F})$.

Duty cycle. The duty cycle was varied between 20,50 and $100 \%$, with an MB/cell ratio of 50:1, a duration of $60 \mathrm{sec}$ and an intensity of $1 \mathrm{~W} / \mathrm{cm}^{2}$. Using a $100 \%$ duty cycle, the cell viability at $24 \mathrm{~h}$ was significantly lower than that immediately following UTMD ( $\mathrm{P}<0.001$; Fig. 1G).

Dosage of SonoVue. The MB/cell ratio varied between 20:1, 50:1 and 70:1 at a duration of $60 \mathrm{sec}$, a duty cycle of $50 \%$ and an intensity of $1 \mathrm{~W} / \mathrm{cm}^{2}$. At an $\mathrm{MB} /$ cell ratio of $70: 1$, cell viability at $24 \mathrm{~h}$ was significantly lower than that at $0 \mathrm{~h}$ post-UTMD ( $\mathrm{P}=0.0281$; Fig. $1 \mathrm{H})$.

US intensity and duration of exposure. US intensity was varied between 1,2 and $3 \mathrm{~W} / \mathrm{cm}^{2}$ with a duration of $60 \mathrm{sec}$. Cell viability at an intensity of $3 \mathrm{~W} / \mathrm{cm}^{2}$ was significantly lower than that at $1 \mathrm{~W} / \mathrm{cm}^{2}(\mathrm{P}<0.001$, Fig. 1I). Intensities of 1,2 and $3 \mathrm{~W} / \mathrm{cm}^{2}$ were applied at a duration of $120 \mathrm{sec}$. Cell viability was shown to decrease in an intensity-dependent manner. Cell viability at an intensity of $3 \mathrm{~W} / \mathrm{cm}^{2}$ was significantly lower than that at 1 or $2 \mathrm{~W} / \mathrm{cm}^{2}(\mathrm{P}<0.001$; Fig. $1 \mathrm{~J})$.

Duty cycle. A duty cycle of $100 \%$ significantly reduced cell viability compared with that in the $20 \%$ and $50 \%$ duty cycle groups $(\mathrm{P}<0.001$; Fig. $1 \mathrm{~K})$. There was no significant difference in the cell viability with duty cycles of $20 \%$ and $50 \%(\mathrm{P}>0.05)$.

Dosage of SonoVue. An MB/cell ratio of 70:1 significantly damaged cells compared with ratios of 20:1 and 50:1 ( $\mathrm{P}<0.001$, respectively; Fig. 1L). There was no significant difference in cell viability between the 20:1 and 50:1 groups ( $P>0.05)$.

\section{Transfection of PEI/pDNA-, L/siRNA- or rAAV of RPE cells using US and/or SonoVue}

Gene transfer by US. The ratio of positively transfected cells and the mean fluorescence density were compared between the different groups (Fig. 2). There were no differences in the ratios of positively transfected cells or the mean 

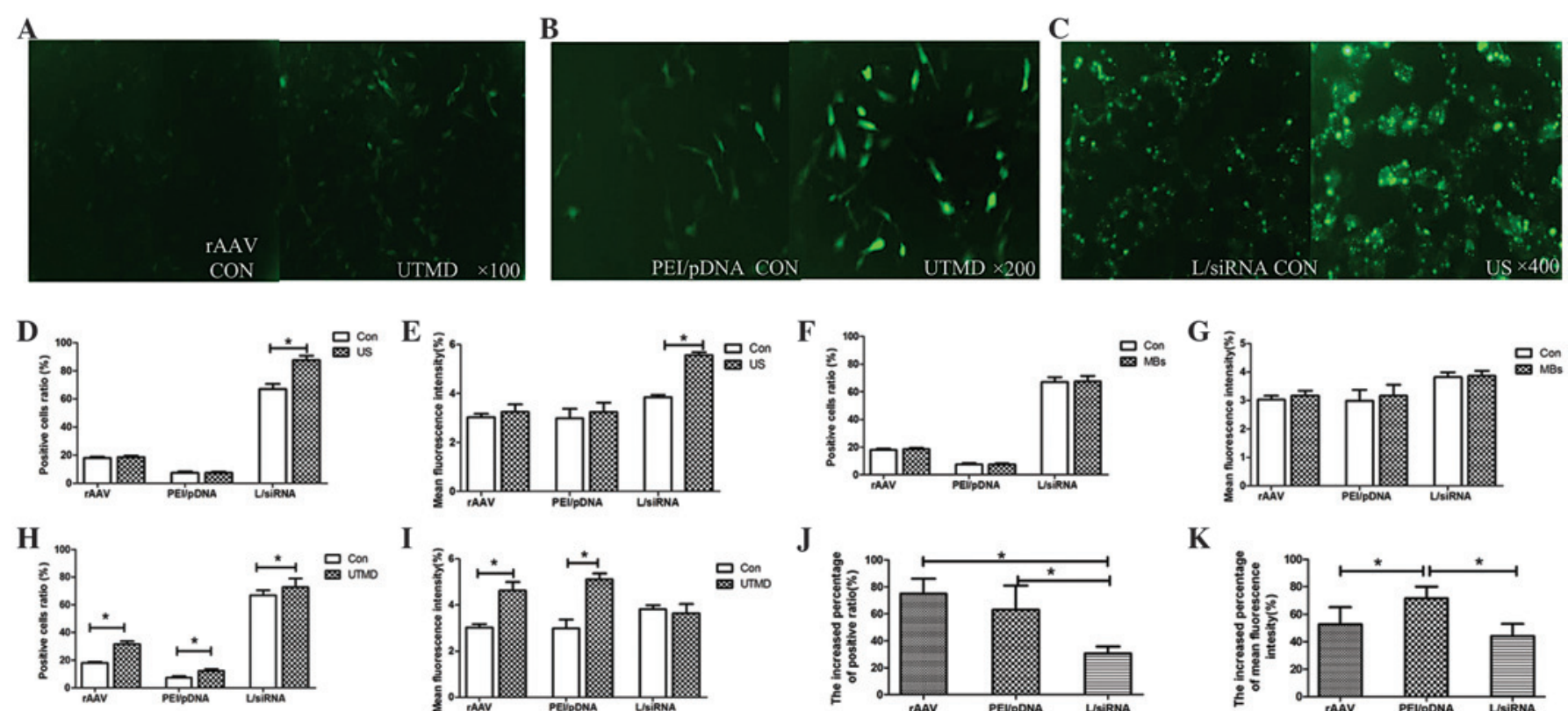

I

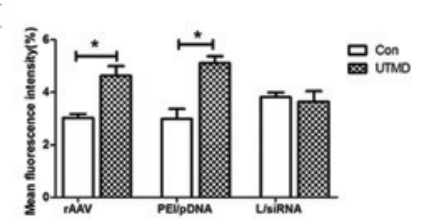

$\mathbf{J}$

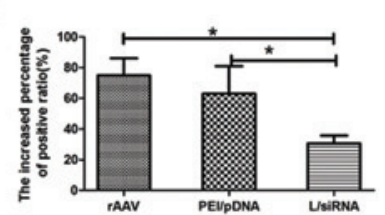

$\mathbf{K}$

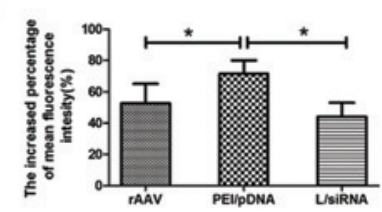

Figure 2. rAAV, PEI/pDNA and L/siRNA transfection mediated by US with or without SonoVue. (C-E) In the L/siRNA group, the ratio of positive cells and mean fluorescence density in the US only group were significantly higher than those in the control group $(\mathrm{P}<0.001) ;(\mathrm{F}, \mathrm{G})$ there were no differences between the SonoVue only groups and the corresponding control groups of rAAV, PEI/pDNA and siRNA. (A, B, H) UTMD significantly enhanced the positive cell ratio compared with that in the corresponding control groups of rAAV, PEI/pDNA and siRNA ( $\mathrm{P}<0.001, \mathrm{P}<0.001$ and 0.0244 , respectively). (A, B, I) The mean fluorescence density was significantly higher than that of the corresponding control groups of rAAV, PEI/pDNA (P<0.001 and 0.0004$)$. (J) The increased positive cell ratio in the UTMD/rAAV and UTMD/ PEI/pDNA groups was significantly higher than that in the US/ L/siRNA group (P<0.001 and 0.001$)$. (K) The increased percentage of mean fluorescence density in the UTMD/PEI/pDNA group was significantly higher than that in the UTMD/rAAV and US/L/siRNA groups ( $\mathrm{P}=0.011$ and 0.009 ). UTMD, ultrasound-targeted microbubble destruction; US, ultrasound; rAAV, type 2 recombinant adeno-associated virus vector encoding the EGFP gene; PEI, polyethylenimine; L/siRNA, lipofectamine/carboxyfluorescein-labeled small interfering RNA; MBs, microbubbles; pDNA, polyethylamine/plasmid enhanced green fluorescent protein-N1; Con, control.

fluorescence densities between the US exposure groups and the corresponding control groups for $\mathrm{rAAV}$ and PEI/pDNA. In the L/siRNA group, the ratio of positively transfected cells and the mean fluorescence density were significantly higher than those of the control group ( $\mathrm{P}<0.001$; Fig. 2D and E).

Gene transfer by SonoVue. There were no significant differences in the percentages of positively transfected cells and the mean fluorescence densities between the SonoVue groups and the corresponding control groups for the $\mathrm{rAAV}, \mathrm{PEI} / \mathrm{pDNA}$ and L/siRNA vectors ( $\mathrm{P}>0.05$ for all; Fig. $2 \mathrm{~F}$ and $\mathrm{G}$ ).

Gene transfer by US and SonoVue. In the rAAV, PEI/pDNA and L/siRNA groups, UTMD significantly enhanced the percentage of positively transfected cells compared with those in the control groups $(\mathrm{P}<0.001, \mathrm{P}<0.001$ and $\mathrm{P}=0.0244$, respectively; Fig. $2 \mathrm{H}$ ). The mean fluorescence density was significantly higher than that of the corresponding control groups for rAAV and $\mathrm{PEI} / \mathrm{pDNA}(\mathrm{P}<0.001$ and $\mathrm{P}=0.0004$, respectively; Fig. 2I).

Comparison of the increased transfection efficiency among UTMD/rAAV, UTMD/PEI/pDNA and US/L/siRNA groups. The increased percentage of positively transfected cells in the $\mathrm{UTMD} / \mathrm{rAAV}$ and UTMD/PEI/pDNA groups was significantly higher than that in the US/L/siRNA group $(\mathrm{P}<0.001$ and $\mathrm{P}=0.001$, respectively; Fig. $2 \mathrm{~J}$ ). The increased percentage of the mean fluorescence density in the UTMD/PEI/pDNA group was significantly higher than that in the UTMD/rAAV and US/L/siRNA groups ( $\mathrm{P}=0.011$ and 0.009 , respectively; Fig. $2 \mathrm{~K}$ ).

Effect of UTMD on pDNA, rAAV, PEI/pDNA and L/siRNA. Agarose gel electrophoresis was used to evaluate the structure
A

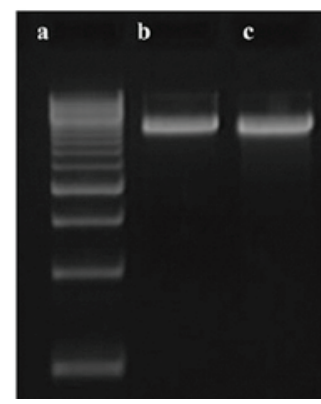

B

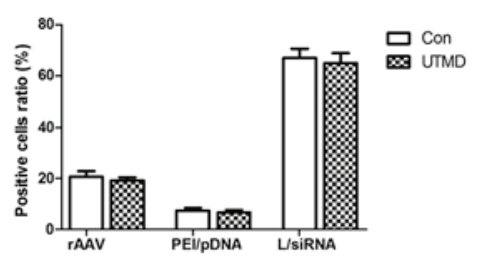

Figure 3. Effect of UTMD on pDNA, rAAV, PEI/pDNA and L/siRNA. (A) Plasmid electrophoresis of the supernatent following UTMD. (a) Marker $5 \mathrm{~kb}$ ladder, (b) pDNA only and (c) UTMD/pDNA. (B) Percentage of positive cells in each of the three groups with and without UTMD. UTMD, ultrasound-mediated microbubble destruction; pDNA, polyethylamine/plasmid enhanced green fluorescent protein-N1; rAAV, type 2 recombinant adeno-associated virus vector encoding the EGFP gene; L/siRNA, lipofectamine/carboxyfluorescein-labeled small interfering RNA.

of pDNA in the supernatant following exposure to UTMD and compared with that without treatment. The migration rate during electrophoresis of the EGFP plasmid remained 
unchanged, with supercoiled DNA remaining dominant in the electrophoresis band, and the optical density of supercoiled DNA in the same lane showing no significant change (Fig. 3A). The effect of UTMD on the transfection efficacy of rAAV, PEI/pDNA and L/siRNA in cells was studied (Fig. 3B). There was no significant difference between the experimental groups and the corresponding control groups $(\mathrm{P}>0.05)$.

\section{Discussion}

Considering the significant morbidity of numerous refractory retinal diseases and the limitations of the available treatment, the advancement of gene therapy is a promising development in this field. RPE maintains the function of the photoreceptors and is an important target in retinal gene delivery. Studies in non-viral gene delivery approaches have reported limited success in the transfection of RPE cells in vitro. One study using solid lipid nanoparticles to transfect ARPE-19 cells only achieved $2.5 \%$ efficacy (4). The most effective reagent identified in another study achieved only $12-15 \%$ efficiency even when it optimized a number of different commercially available reagents for the transfection of ARPE-19 cells (6). A further study into the transfection of RPE cells reported $<1 \%$ for Lipofectin, 1-3\% for DOTAP/DOGS and $<5 \%$ using degraded dendrimers (5). RPE cells are considered a cell type which is difficult to be transfected $(3-6,15)$. A novel method of delivery is thus required to augment the efficiency of gene transfer to ARPE-19 cells.

pDNA or siRNA are degraded enzymatically in the body, and UTMD remains to have a low proficiency in promoting transfection (16-18). In order to enhance the efficiency of transfection, UTMD may be combined with other transfection methods. UTMD has been shown to enhance liposomal-, PEIand adeno-associated virus-mediated gene transfer in vitro and in vivo (16-18). To the best of our knowledge there is no information on UTMD-mediated non-viral vector transfection of RPE cells. In the present study, the efficiency of UTMD combined with $\mathrm{PEI} / \mathrm{pDNA}, \mathrm{L} / \mathrm{siRNA}$ and $\mathrm{rAAV}$ in promoting transfection in RPE cells was investigated.

To establish the optimal conditions for UTMD-mediated gene transfer to RPE cells, the effects of various conditions on cell viability at 0 and $24 \mathrm{~h}$ following UTMD were examined. Through the vibration and burst of microbubbles, ultrasonic cavitation produces a mechanical shearing force, forms free radicals, increases the intracellular calcium concentration and causes damage to the mitochondrial membrane (16-19). The series of sonochemical reactions produced may cause the loss of function, dissolution or death of cells. The present study showed that the majority of cells did not die immediately following UTMD, but that UTMD affected the microstructure inside the cells, and thus inhibited cell proliferation. Therefore, cell viability at $24 \mathrm{~h}$ was lower than that immediately following UTMD. Cell viability at $24 \mathrm{~h}$ following UTMD using various exposure parameters was also investigated. Different UTMD parameters may have different effects on the cells, possibly due to certain irradiation parameters leading to cell membrane fatigue, so that the corresponding position of the cell membrane was damaged. The results indicated that cell viability evaluation at $24 \mathrm{~h}$ post-UTMD provided a more accurate result than immediate assessment following UTMD. In addition, an
US intensity of $3 \mathrm{~W} / \mathrm{cm}^{2}$, a $100 \%$ duty cycle and an $\mathrm{MB} /$ cell ratio of 70:1 caused significant damage to the cells and should therefore not be used in future investigations. Furthermore, the combination of parameters used in the transfection experiments was safe as cell viability at $24 \mathrm{~h}$ was $97.88 \%$.

A preliminary study by our group produced an optimized method for the delivery of rAAV (12-14), PEI/pDNA and L/siRNA into RPE cells, including the optimized US exposure parameters, the N/P ratio and the dosage of $\mathrm{L}$ and siRNA (data not shown). The present study evaluated the efficiency (as measured by the percentage of positively transfected cells and the mean fluorescence density) of UTMD combined with $\mathrm{PEI} / \mathrm{pDNA}, \mathrm{L} / \mathrm{siRNA}$ and $\mathrm{rAAV}$ in enhancing transfection in RPE cells. The results showed that $\mathrm{rAAV}$ and PEI/pDNA with US exposure alone did not increase transfection efficiency, whereas US markedly increased L/siRNA transfection efficiency. The majority of studies have shown that USMT enhanced gene transfer efficiency. However, in the present study, USMT did not increase the transfer efficiency of rAAV and PEI/pDNA. One study that investigated the mechanisms by which UTMD increases AAV transfection suggested that sonoporation enabled AAV to gain direct access to the cell cytoplasm (19). The energy of USMT, as opposed to that of UTMD, may be insufficient for the formation of sonoporation in RPE cells. USMT was shown to increase L/siRNA transfection efficiency. At lower ultrasound energy levels, oscillation occurs in the vicinity of cells and gently agitates the cell membrane. This phenomenon has been demonstrated in different studies and the process is not known to induce endocytosis $(20,21)$. In order for the transfection of liposomes to occur, they fuse with the plasma membrane and enter the cell by endocytosis $(20,21)$. It is possible that the endocytosis generated by the lower ultrasound intensities produces a synergistic effect with the self-transfer of liposomes. PEI has a high density of positive charges and has been shown to interact with the negatively charged cell membrane and enter the cell through endocytosis. USMT does not produce a synergistic effect with the endocytosis of PEI.

The present study showed that rAAV, pDNA and L/siRNA in combination with MBs, but without US, did not increase transfection efficiency. One study on the comparison between the effect of different microbubble contrast agents on gene transfection showed that Optison improved pDNA transfection efficiency into skeletal muscles of mice but that SonoVue and Levovist did not (22). A previous study also found that SonoVue alone enhanced rAAV transfection efficiency to RPE-J cells (14). The nonspecific phagocytic ability of cells to phagocytize MBs may be the key factor underlying this phenomenon.

UTMD significantly increased rAAV and PEI/pDNA transfer to human RPE cells. MBs acting as cavitation nuclei effectively focus US energy and potentiate further bioeffects (23). Therefore, using UTMD, the energy produced is sufficient for the formation of transient pores in ARPE-19 cells. Although UTMD significantly increased L/siRNA transfection, its efficiency was lower than that of US exposure alone. Endocytosis induced by the low energy of US alone was more effective in L/siRNA transfection than sonoporation induced by the higher energy UTMD .

UTMD/US enhanced the transfection efficiency of rAAV, PEI/pDNA and L/siRNA by increasing the transgene expression per cell and the percentage of transfected cells. 
UTMD-mediated rAAV or PEI/pDNA delivery was more effective than that of L/siRNA. In the present study, the percentage of positively transfected cells increased to $74.88 \pm 11.03 \%$ and transgene expression per cell increased to $71.46 \pm 8.58 \%$. In RPE cells, higher transgene expression per cell was more important than higher percentages of positive cells. Due to the small size of the retinal cells and short distance between RPE and the neural retina, a small number of transfected RPE cells may provide sufficient levels of a potent secreted protein to produce successful retinal gene therapy (24). A very small number of transfected cells $(<0.1 \%)$ was adequate for therapeutic efficacy of secreted gene product, vascular endothelial growth factor, in the vascular wall (25).

The effect of different UTMD parameters on the transfection ability of rAAV, PEI/pDNA and L/siRNA in cells was also evaluated. No significant difference was detected between the exposure groups and the control groups. Thus, UTMD had no apparent effect on the transfection ability of rAAV, PEI/pDNA and L/siRNA. Furthermore, agarose gel electrophoresis showed that UTMD did not alter the structure of pDNA in the supernatant.

In conclusion, the present study demonstrated that UTMD/US may be used to enhance rAAV, PEI/pDNA and L/siRNA transfection efficiency of ARPE-19 cells in vitro. Studies on different genes (including pDNA and siRNA) and different types of vectors (chemical and biological) mediated by UTMD may provide useful information for future in vivo and transfection studies.

\section{Acknowledgements}

This study was supported by the National Natural Science Foundation of China (grant no. 81200700) and the Natural Science Foundation of Shanghai (grant no. 11ZR1421100).

\section{References}

1. Algvere PV, Berglin L, Gouras P and Sheng Y: Transplantation of fetal retinal pigment epithelium in age-related macular degeneration with subfoveal neovascularization. Graefes Arch Clin Exp Ophthalmol 232: 707-716, 1994.

2. Li LX and Turner JE: Inherited retinal dystrophy in the RCS rat: prevention of photoreceptor degeneration by pigment epithelial cell transplantation. Exp Eye Res 47: 911-917, 1988.

3. Sunshine JC, Sunshine SB, Bhutto I, Handa JT and Green JJ: Poly( $\beta$-amino ester)-nanoparticle mediated transfection of retinal pigment epithelial cells in vitro and in vivo. PLoS One 7: e37543, 2012.

4. del Pozo-Rodriguez A, Delgado D, Solinis MA, Gascón AR and Pedraz JL: Solid lipid nanoparticles for retinal gene therapy: transfection and intracellular trafficking in RPE cells. Int J Pharm 360: 177-183, 2008.

5. Urtti A, Polansky J, Lui GM and Szoka FC: Gene delivery and expression in human retinal pigment epithelial cells: effects of synthetic carriers, serum, extracellular matrix and viral promoters. J Drug Target 7: 413-421, 2000.

6. Abul-Hassan K, Walmsley R and Boulton M: Optimization of non-viral gene transfer to human primary retinal pigment epithelial cells. Curr Eye Res 20: 361-366, 2000.

7. Fechheimer M, Boylan JF, Parker S, Sisken JE, Patel GL and Zimmer SG: Transfection of mammalian cells With plasmid DNA by scrape loading and sonication loading. Proc Natl Acad Sci USA 84: 8463-8467, 1987.
8. Zhou XY, Liao Q, Pu YM, Tang YQ, Gong X, Li J, Xu Y and Wang ZG: Ultrasound-mediated microbubble delivery of pigment epithelium-derived factor gene into retina inhibits choroidal neovascularization. Chin Med J 22: 2711-2717, 2009.

9. Sonoda S, Tachibana K, Yamashita T, Shirasawa M, Terasaki H, Uchino E, Suzuki R, Maruyama K and Sakamoto T: Selective gene transfer to the retina using intravitreal ultrasound irradiation. J Ophthalmol 12: 412752, Epub 2012.

10. Sanders NN, Peeters L, Lentacker I, Demeester J and De Smedt SC: Wanted and unwanted properties of surface PEGylated nucleic acid nanoparticles in ocular gene transfer. J Control Release 122: 226-235, 2007.

11. Xie W, Liu S, Su H, Wang Z, Zheng Y and Fu Y: Ultrasound microbubbles enhance recombinant adeno-associated virus vector delivery to retinal ganglion cells in vivo. Acad Radiol 17: 1242-1248, 2010.

12. Li HL, Zheng XZ, Wang HP, Li F, Wu Y and Du LF: Ultrasound-targeted microbubble destruction enhances AAV-mediated gene transfection in human RPE cells in vitro and rat retina in vivo. Gene Ther 16:1146-1153, 2009.

13. Zheng XZ, Wu Y, Li HL, Du LF, Wang HP and Gu Q: Comparative analysis of the effects of ultrasound-targeted microbubble destruction on recombinant adeno-associated virus- and plasmid-mediated transgene expression in human retinal pigment epithelium cells. Mol Med Rep 2: 937-942, 2009.

14. Zheng XZ, Li HL, Du LF, Wang HP and Gu Q: Comparative analysis of gene transfer to human and rat retinal pigment epithelium cell line by a combinatorial use of recombinant adeno-associated virus and ultrasound or/and microbubbles. Bosn J Basic Med Sci 9: 174-181, 2009.

15. Mannermaa E, Rönkkö S, Ruponen M, Reinisalo M and Urtti A: Long-lasting secretion of transgene product from differentiated and filter-grown retinal pigment epithelial cells after nonviral gene transfer. Curr Eye Res 30: 345-353,2005.

16. Müller OJ, Schinkel S, Kleinschmidt JA, Katus HA and Bekeredjian R: Augmentation of AAV-mediated cardiac gene transfer after systemic administration in adult rats. Gene Ther 15: 1558-1565, 2008.

17. Zhang CB, Cao HL, Li Q, Tu J, Guo X, Liu Z and Zhang D: Enhancement effect of ultrasound-induced microbubble cavitation on branched polyethylenimine-mediated VEGF (165) transfection with varied N/P ratio. Ultrasound Med Biol 39: 161-171, 2013.

18. Afadzi M, Strand SP, Nilssen EA, Måsøy SE, Johansen TF, Hansen R, Angelsen BA and de L Davies C: Mechanisms of the ultrasound-mediated intracellular delivery of liposomes and dextrans. IEEE Trans Utrason Ferroelectr Freq Control 60: 21-33, 2013.

19. Geers B, Lentacker I, Alonso A, Sanders NN, Demeester J, Meairs S and De Smedt SC: Elucidating the mechanisms behind sonoporation with adeno-associated virus-loaded microbubbles. Mol Pharm 8: 2244-2251, 2011.

20. Lee JL, Lo CW, Ka SM, Chen A and Chen WS: Prolonging the expression duration of ultrasound-mediated gene transfection using PEI nanoparticles. J Control Release 160: 64-71, 2012.

21. Juffermans LJ, Meijering BD, Henning RH and Deelman LE: Ultrasound and microbubble-targeted delivery of small interfering RNA into primary endothelial cells is more effective than delivery of plasmid DNA. Ultrasound Med Biol 40: 532-40, 2014.

22. Wang X, Liang HD, Dong B, Lu QL and Blomley MJ: Gene transfer with microbubble ultrasound and plasmid DNA into skeletal muscle of mice: comparison between commercially available microbubble contrast agents. Radiology 237: 224-229, 2005.

23. Okada K, Kudo N, Niwa K and Yamamoto K: A basic study on sonoporation with microbubbles exposed to pulsed ultrasound. J Med Ultrasonics 32: 3-11, 2005.

24. Haensler J and Szoka FC Jr: Polyamidoamine cascade polymers mediate efficient transfection of cells in culture. Bioconjug Chem 4: 372-379, 1993.

25. Laitinen M, Zachary I, Breier G, et al: VEGF gene transfer reduces intimal thickening via increased production of nitric oxide in carotid arteries. Hum Gene Ther 8:1737-1744, 1997. 